PHYSICAL REVIEW FLUIDS 1, 050505 (2016)

\title{
Merger of a bubble and a soap film
}

\author{
Daniel M. Harris, ${ }^{1,2}$ Giuseppe Pucci, ${ }^{2}$ Victor Prost, ${ }^{2}$ \\ Julio Quintela Casal, ${ }^{2}$ and John W. M. Bush ${ }^{2, *}$ \\ ${ }^{1}$ Department of Mathematics, University of North Carolina at Chapel Hill, \\ Chapel Hill, North Carolina 27599, USA \\ ${ }^{2}$ Department of Mathematics, Massachusetts Institute of Technology, \\ Cambridge, Massachusetts 02139, USA
}

(Received 9 August 2016; published 12 September 2016)

\begin{abstract}
This paper is associated with a video winner of a 2015 APS/DFD Milton van Dyke Award for work presented at the DFD Gallery of Fluid Motion. The original video is available from the Gallery of Fluid Motion, http://dx.doi.org/10.1103/APS.DFD.2015.GFM.V0087
\end{abstract}

DOI: 10.1103/PhysRevFluids.1.050505

The coalescence of a drop into a fluid bath is a subject of continuing interest in fluid dynamics [1,2], owing to its dynamical complexity, its accessibility in the laboratory, and its aesthetic appeal. It has been known for some time that a drop gently placed on a static bath of the same fluid may initially combine only partially with the underlying bath, resulting in a daughter droplet of approximately half the diameter of the parent droplet [3]. This process can then repeat, giving rise to the so-called "coalescence cascade" until the final droplet is small enough to be completely absorbed.

In the present work, we considered a related system, a soap bubble settling onto a planar soap film, and demonstrate that partial coalescence is also possible in this scenario. The partial coalescence of a soap bubble was first predicted by recent numerical simulations [4] and subsequently observed in experiment [5]. As evidenced in Fig. 1, the sequence of events arising when a droplet or bubble undergoes partial coalescence is remarkably similar, despite the very different length scales. Capillary waves generated at the onset of coalescence sweep up the sides of the drop or bubble, delaying complete coalescence and leading to the pinch-off of a daughter drop or bubble of approximately half the diameter of its parent [5,6]. In both scenarios, capillary overpressure drives flow out of the drop or bubble which is principally resisted by the fluid's inertia $[3,5]$; thus, the evacuation process occurs over the interial-capillary time scale $\tau_{\sigma}=\sqrt{\frac{\rho R^{3}}{\sigma}}$, where $\rho$ is the density of the interior fluid, $R$ the radius of the parent drop/bubble, and $\sigma$ the surface tension.

In Fig. 2, we repeat the partial coalescence experiment with a smoke-filled bubble. Following coalescence, a coherent vortex ring is generated by the rapid expulsion of air from the bubble interior. A similar vortex structure arises when a droplet coalesces with a bath [7]. Coalescence cascades up to three steps have been observed in the bubble-film system as depicted in Fig. 3. Further details on the merger of a soap bubble with a soap film are reported elsewhere [5].

\footnotetext{
*bush@math.mit.edu

Published by the American Physical Society under the terms of the Creative Commons Attribution 3.0 License. Further distribution of this work must maintain attribution to the author(s) and the published article's title, journal citation, and DOI.
} 

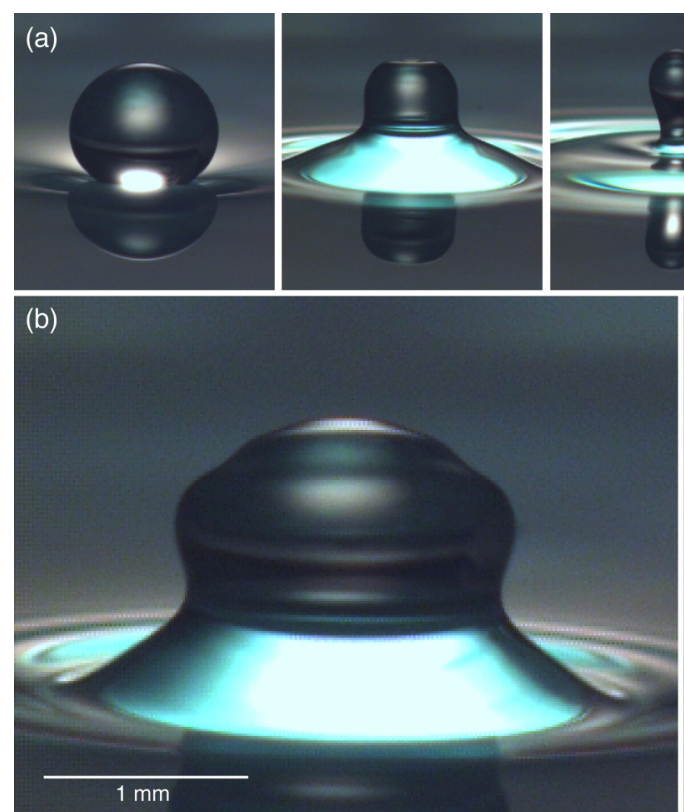

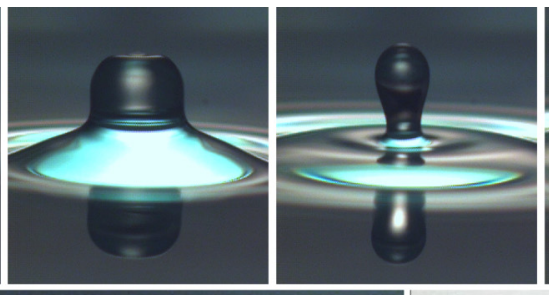

(c)
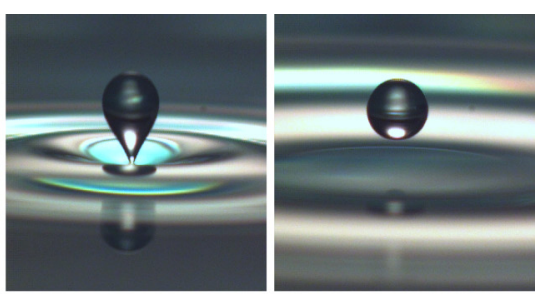
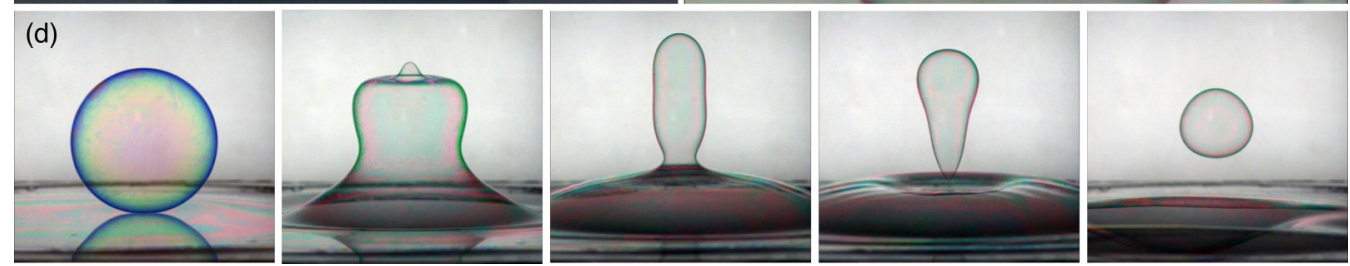

FIG. 1. Comparison of the partial coalescence process of (a-b) a soapy water drop with a bath, and (c-d) a soap bubble with a planar soap film. (a) Evolution from parent to daughter droplet. (d) Evolution from parent to daughter bubble. Comparison of (b) drop and (c) bubble shapes at an intermediate stage. Notice the difference length scales.
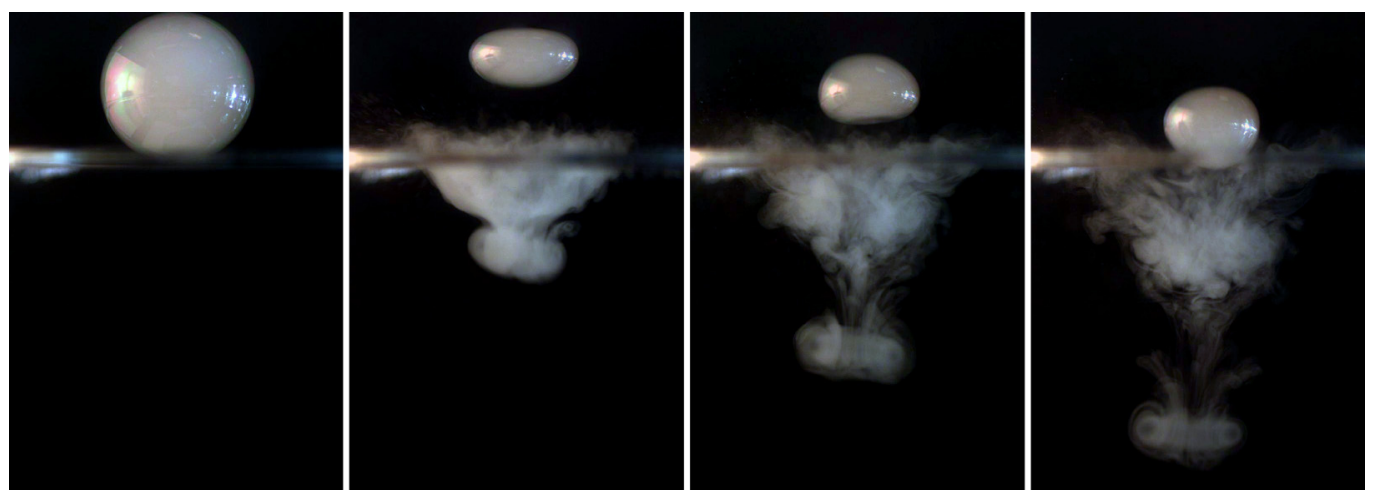

FIG. 2. Flow visualization of the partial coalescence of a smoke-filled soap bubble with a planar soap film. The experiment proceeds from left to right, starting with the initial smoke-filled bubble resting on a soap film. In this particular experiment, the planar soap film ruptures following the partial coalescence process. 


\section{MERGER OF A BUBBLE AND A SOAP FILM}
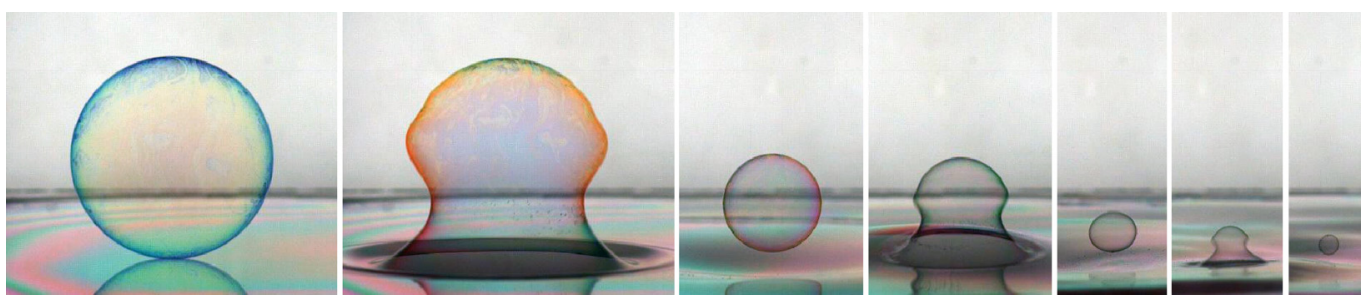

FIG. 3. Soap bubble undergoing three steps of a coalescence cascade from left to right. Following each step, the diameter of the daughter bubble is approximately half that of its parent. DOI: http://dx.doi.org/10.1103/APS.DFD.2015.GFM.V0087

The authors thank the NSF for support through Grant No. CMMI-1333242, and the MIT-France Program.

[1] H. P. Kavehpour, Coalescence of drops, Annu. Rev. Fluid Mech. 47, 245 (2015).

[2] F. H. Zhang, M.-J. Thoraval, S. T. Thoroddsen, and P. Taborek, Partial coalescence from bubbles to drops, J. Fluid Mech. 782, 209 (2015).

[3] S. T. Thoroddsen and K. Takehara, The coalescence cascade of a drop, Phys. Fluids 12, 1265 (2000).

[4] D. W. Martin and F. Blanchette, Simulations of surfactant effects on the dynamics of coalescing drops and bubbles, Phys. Fluids 27, 012103 (2015).

[5] G. Pucci, D. M. Harris, and J. W. M. Bush, Partial coalescence of soap bubbles, Phys. Fluids 27, 061704 (2015).

[6] F. Blanchette and T. P. Bigioni, Partial coalescence of drops at liquid interfaces, Nat. Phys. 2, 254 (2006).

[7] T. Gilet, K. Mulleners, J. P. Lecomte, N. Vandewalle, and S. Dorbolo, Critical parameters for the partial coalescence of a droplet, Phys. Rev. E 75, 036303 (2007). 